\title{
A Structured Approach to Cope with Impurities During Industrial Crystallization Development
}

\author{
Supplementary Information
}

Stephanie J. Urwin, Guillaume Levilain, Ivan Marziano, Jeremy M. Merritt, Ian Houson and Joop H. ter Horst*

Email: joop.terhorst@strath.ac.uk

\section{Contents}

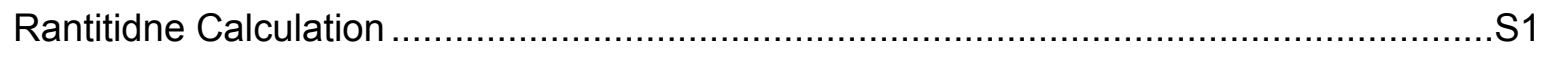

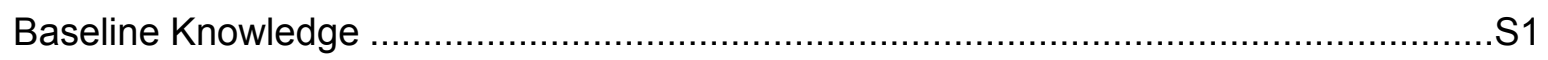

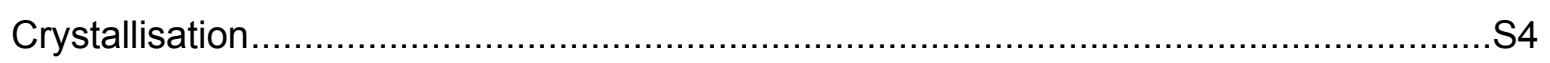

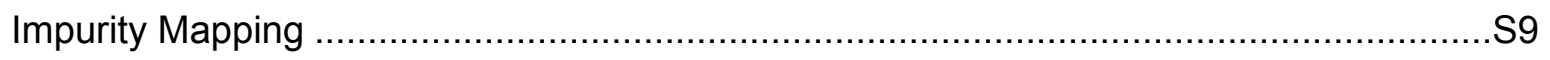

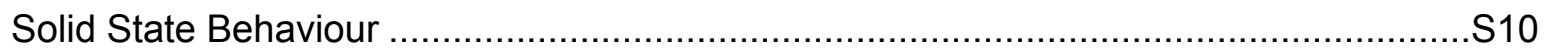

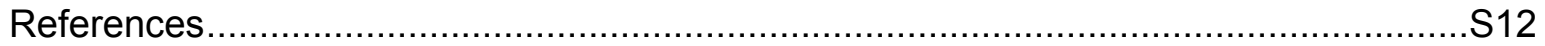




\section{Ranitidine Calculation}

Table S1 Example calculation of crystallisation selectivity in the case of ranitidine hydrochloride and $N$ nitrosodimethylamine

\begin{tabular}{ccc} 
& Ranitidine Hydrochloride & N-Nitrosodimethylamine \\
\hline Molecular Formula & $\mathrm{C}_{13} \mathrm{H}_{22} \mathrm{~N}_{4} \mathrm{O}_{3} \mathrm{~S} \cdot \mathrm{HCl}$ & $\mathrm{C}_{2} \mathrm{H}_{6} \mathrm{~N}_{2} \mathrm{O}$ \\
Molecular Weight & $350.87 \mathrm{~g} \mathrm{~mol}^{-1}$ & $74.083 \mathrm{~g} \mathrm{~mol}^{-1}$ \\
Mass in Feed & $800 \mathrm{mg}$ & $9.6 \times 10^{-4} \mathrm{mg}$ \\
Moles in Feed & $2.28 \mathrm{mmol}$ & $1.30 \times 10^{-5} \mathrm{mmol}$ \\
Mass in Crystals & $599.9 \mathrm{mg}$ & $9.6 \times 10^{-5} \mathrm{mg}$ \\
Moles in Crystals & $1.71 \mathrm{mmol}$ & $1.30 \times 10^{-6} \mathrm{mmol}$ \\
Mass in Mother Liquor & $199.1 \mathrm{mg}$ & $8.64 \times 10^{-4} \mathrm{mg}$ \\
Moles in Mother Liquor & $0.57 \mathrm{mmol}$ & $1.17 \times 10^{-5} \mathrm{mmol}$ \\
Distribution Coefficient & $K_{A}=1.0000$ & $K_{i}=0.0378$ \\
Selectivity & & $\mathbf{\alpha}=\mathbf{0 . 0 3 8}$ \\
\hline
\end{tabular}

This calculation assumes that impurity $\mathrm{N}$-nitrosodimethylamine is present in the crystallisation feed and is not formed during the crystallisation process. Ranitidine is tableted as the hydrochloride salt.

\section{Baseline Knowledge}

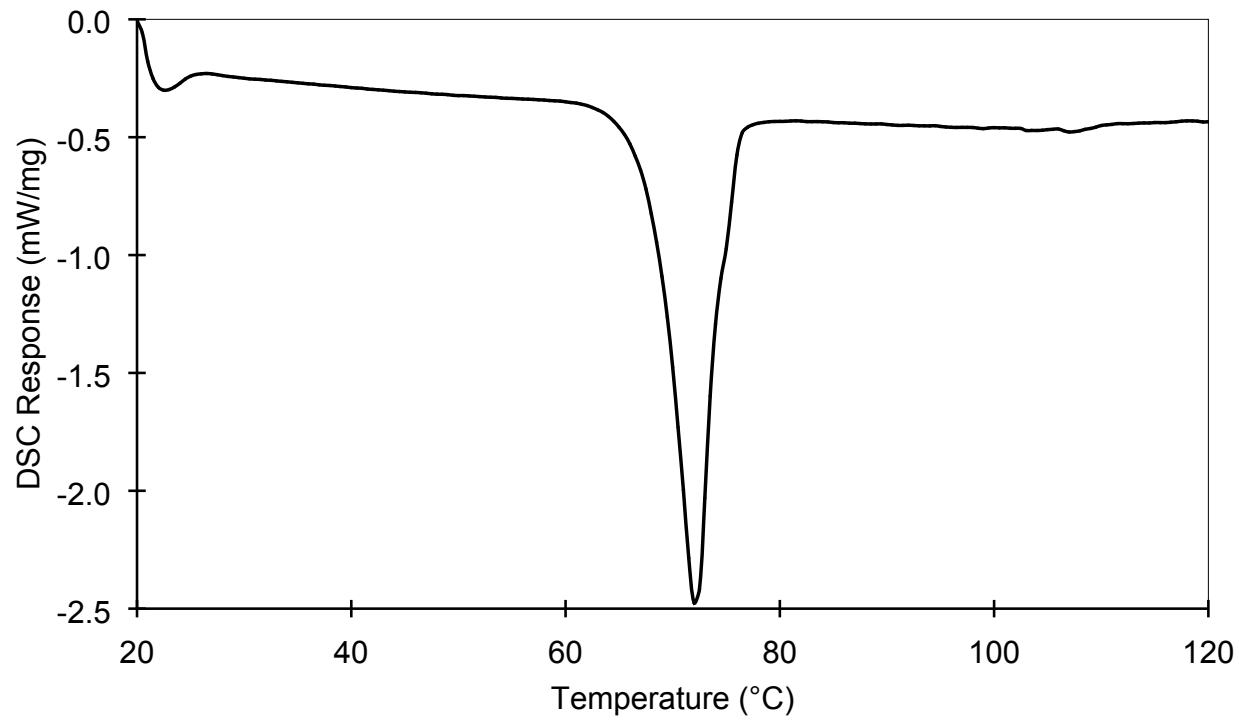

Figure S1 DSC analysis of synthesised Impurity E. Peak onset $=66.4^{\circ} \mathrm{C}$, peak maximum $=72.1^{\circ} \mathrm{C}$, peak area $=$ $61.9 \mathrm{~J} / \mathrm{g}$. 
Table S2 Physical properties of compounds used in the example crystallisations

\begin{tabular}{cccccc}
\hline Compound & Mw & \multicolumn{2}{c}{ Polymorphs } & $\mathbf{T}_{\mathbf{m}}\left({ }^{\circ} \mathbf{C}\right)$ & $\begin{array}{c}\mathbf{H}_{\text {fus }}(\mathbf{k J} \\
\left.\mathbf{m o l}^{-1}\right)\end{array}$ \\
\hline Paracetamol & 151.2 & I & HXACAN & 163 & 27.6 \\
& & II & HXACAN08 & 159 & \\
Acetanilide & 114.3 & III & HXACAN39 & 143 & \\
Metacetamol & 151.2 & I & MEANIL & & 21.4 \\
& & MENSEE & 146.9 & 23.0 \\
Fenofibrate & 360.8 & I & MENSEE04 & 125.8 & \\
& & II & TADLIU & 82.5 & 29.9 \\
Fenofibric Acid & 318.8 & I & QANHUJ & 185.5 & 40.7 \\
Impurity E & 346.8 & I & PAWFUQ & 72.1 & 21.5 \\
\hline
\end{tabular}
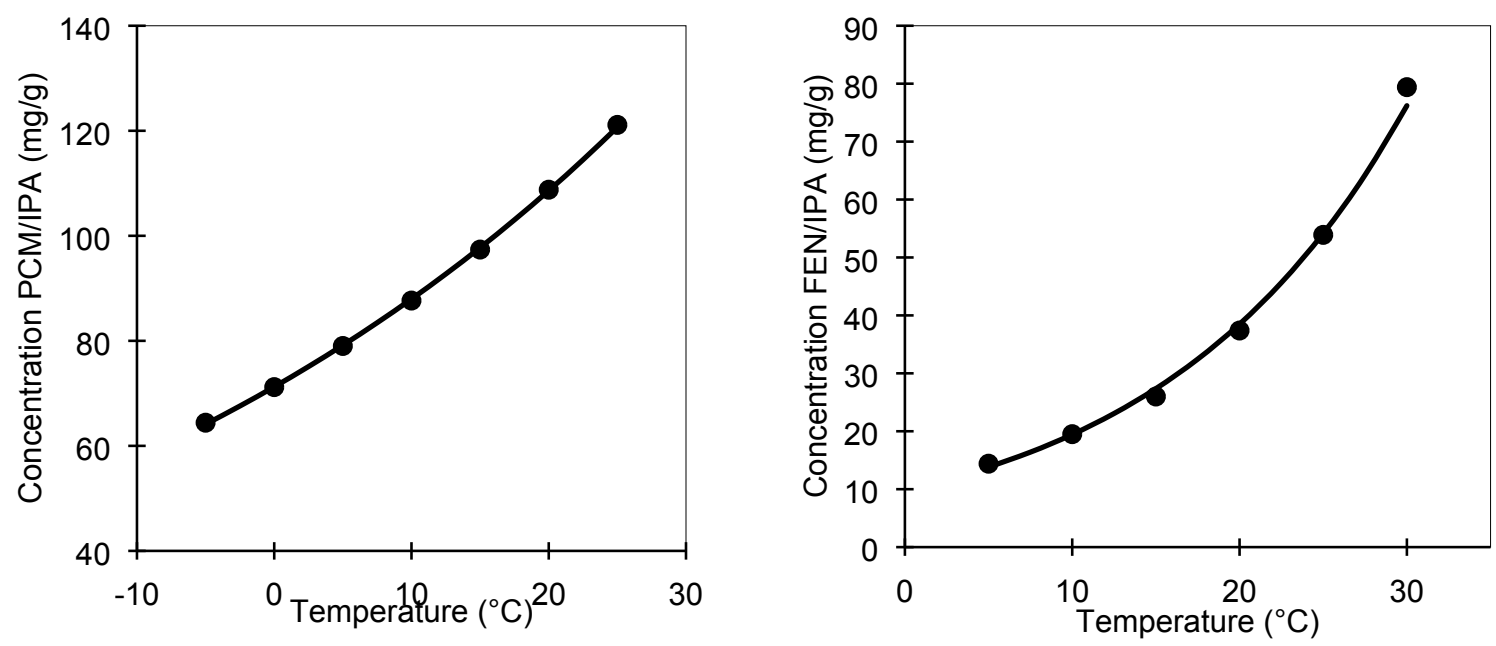

Figure S2 Literature reported gravimetric solubility of paracetamol ${ }^{1}\left(a, y=71.25 \mathrm{e}^{0.021 x}, R^{2}=0.999\right)$ and fenofibrate ${ }^{2}$ $\left(b, y=9.82 e^{0.068 x}, R^{2}=0.997\right)$ in 2-propanol used for cooling crystallisations in stage 2

Table S3 Summary of HPLC parameters and retention times for API and impurities

\begin{tabular}{cccc}
\hline Compound & Sample Phase & Mobile Phase & Retention Time (min) \\
\hline Paracetamol & $5 \% \mathrm{MeOH}, 95 \% \mathrm{H}_{2} \mathrm{O}$ & $20 \% \mathrm{MeOH}, 80 \% \mathrm{H}_{2} \mathrm{O}$ & 1.487 \\
Acetanilide & $5 \% \mathrm{MeOH}, 95 \% \mathrm{H}_{2} \mathrm{O}$ & $20 \% \mathrm{MeOH}, 80 \% \mathrm{H}_{2} \mathrm{O}$ & 5.034 \\
Metacetamol & $5 \% \mathrm{MeOH}, 95 \% \mathrm{H}_{2} \mathrm{O}$ & $20 \% \mathrm{MeOH}, 80 \% \mathrm{H}_{2} \mathrm{O}$ & 2.123 \\
Fenofibrate & $\mathrm{MeCN}$ & $20 \% \mathrm{H}_{2} \mathrm{O}(\mathrm{pH} 3), 80 \% \mathrm{MeCN}$ & 2.675 \\
Fenofibric Acid & $\mathrm{MeCN}$ & $20 \% \mathrm{H}_{2} \mathrm{O}(\mathrm{pH} 3), 80 \% \mathrm{MeCN}$ & 1.223 \\
Impurity E & $\mathrm{MeCN}$ & $20 \% \mathrm{H}_{2} \mathrm{O}(\mathrm{pH} 3), 80 \% \mathrm{MeCN}$ & 2.171 \\
\hline
\end{tabular}


(a)

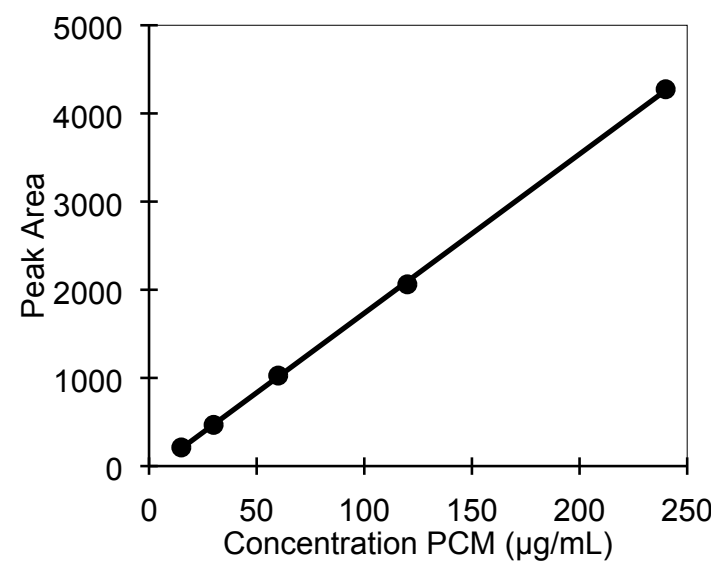

(c)

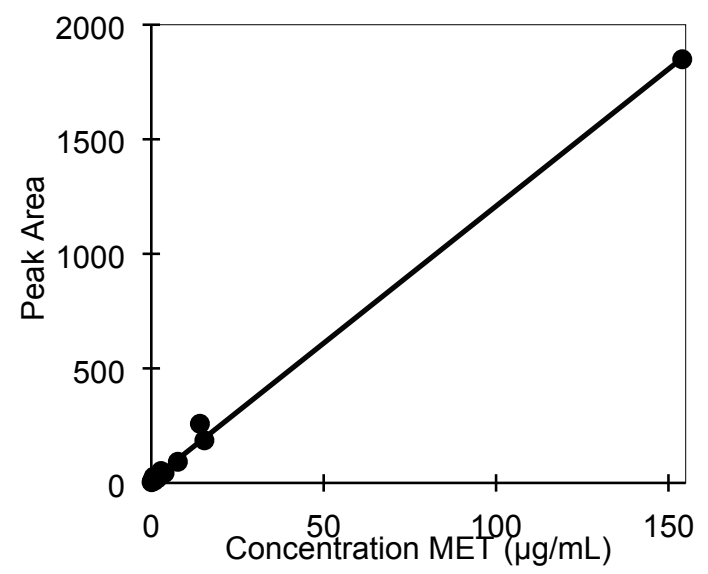

(e)

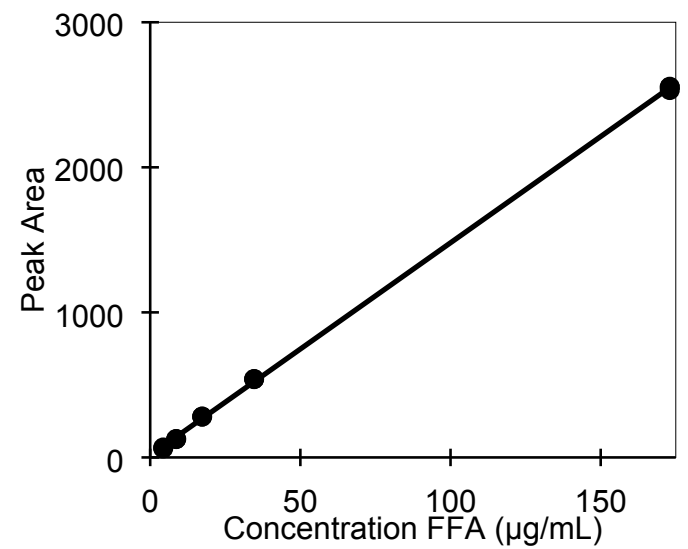

(b)

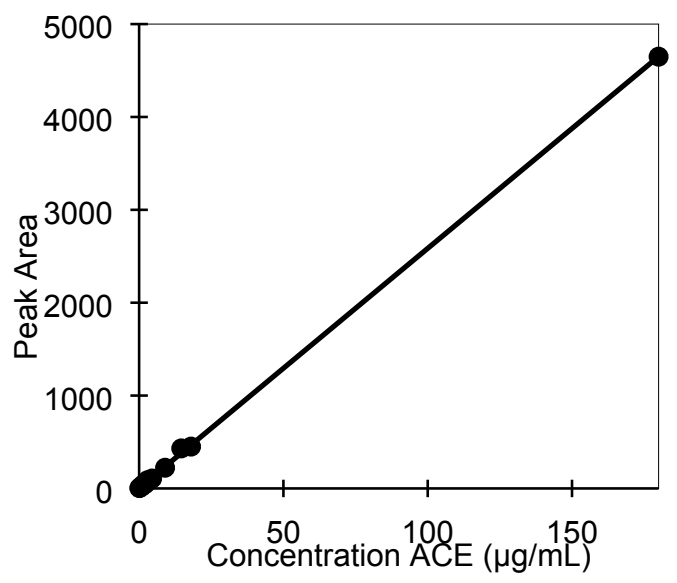

(d)

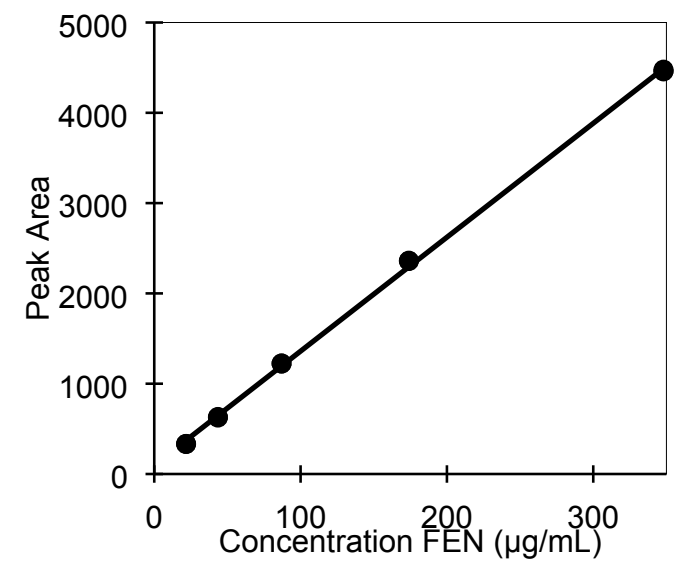

(f)

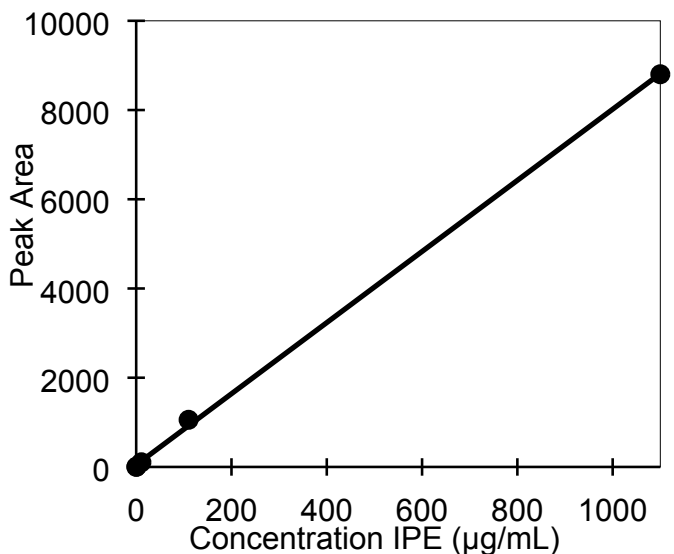

Figure S3 HPLC calibrations for paracetamol $\left(a, y=18.045 x-69.98, R^{2}=0.999\right)$, acetanilide $(b, y=25.819 x+$ $\left.3.12, R^{2}=0.999\right)$, metacetamol $\left(c, y=11.965 x+11.74, R^{2}=0.997\right)$, fenofibrate $\left(d, y=12.619 x+98.39, R^{2}=\right.$ $0.999)$, fenofibric acid $\left(e, y=14.651 x+14.78, R^{2}=1.000\right)$ and impurity $E\left(f, y=7.971 x+46.65, R^{2}=0.999\right)$ used here to measure concentrations of solid and liquid phases. 


\section{Crystallisation}

Table S4 Calculation of maximum selectivity value for fenofibrate and fenofibric acid case study.

\begin{tabular}{|c|c|c|}
\hline & Fenofibrate & Fenofibric Acid \\
\hline Molecular Formula & $\mathrm{C}_{20} \mathrm{H}_{21} \mathrm{ClO}_{4}$ & $\mathrm{C}_{17} \mathrm{H}_{15} \mathrm{ClO}_{4}$ \\
\hline Molecular Weight & $360.831 \mathrm{~g} \mathrm{~mol}^{-1}$ & $318.75 \mathrm{~g} \mathrm{~mol}^{-1}$ \\
\hline $\mathbf{X f}$ & 0.929 & 0.071 \\
\hline Xs & 0.980 & 0.020 \\
\hline $\mathbf{X I}$ & 0.687 & 0.313 \\
\hline $\begin{array}{l}\text { Distribution Coefficient } \\
\text { Selectivity Coefficient }\end{array}$ & 1.427 & 0.447 \\
\hline
\end{tabular}

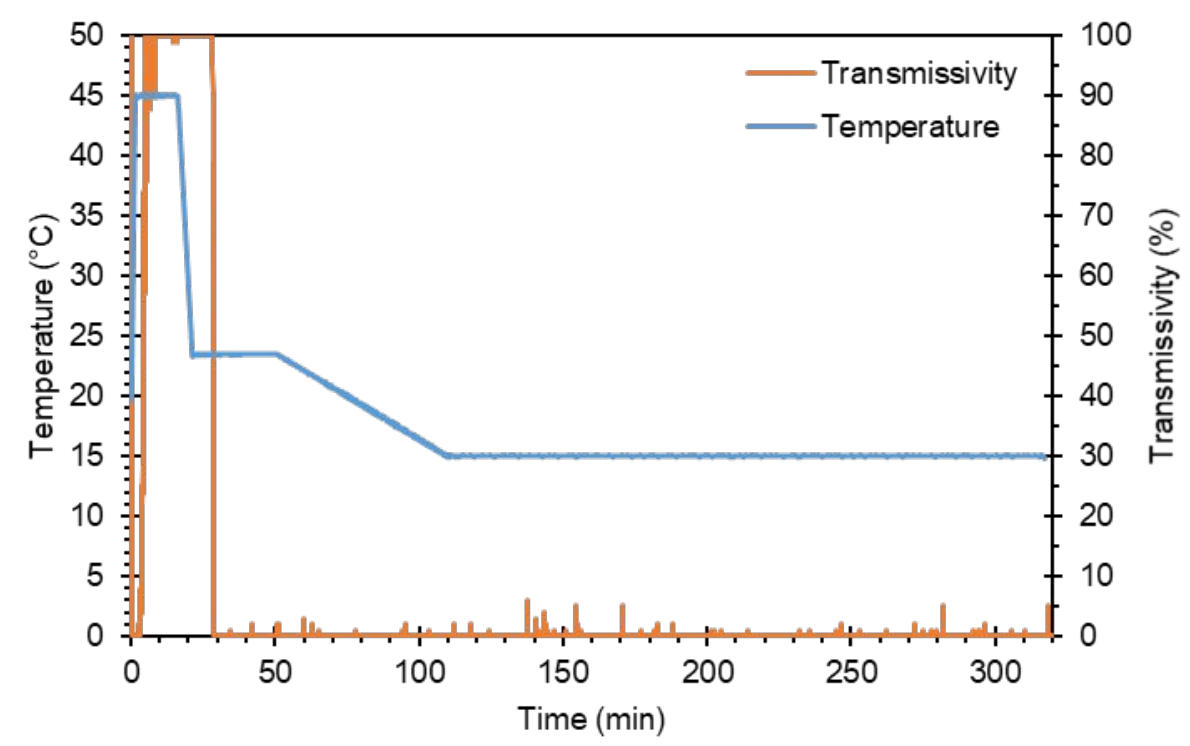

Figure S4 Temperature and transmissivity profile for fenofibrate-fenofibric acid crystallisation. Time to crystallisation was $6 \mathrm{~min} 40 \mathrm{sec}$.

Table S5 Calculation of maximum selectivity value for fenofibrate and impurity E case study.

\begin{tabular}{|c|c|c|}
\hline & Fenofibrate & Impurity E \\
\hline Molecular Formula & $\mathrm{C}_{20} \mathrm{H}_{21} \mathrm{ClO}_{4}$ & $\mathrm{C}_{19} \mathrm{H}_{19} \mathrm{ClO}_{4}$ \\
\hline Molecular Weight & $360.831 \mathrm{~g} \mathrm{~mol}^{-1}$ & $346.8 \mathrm{~g} \mathrm{~mol}^{-1}$ \\
\hline $\mathbf{X f}$ & 0.913 & 0.087 \\
\hline Xs & 0.998 & 0.002 \\
\hline $\mathbf{X I}$ & 0.518 & 0.482 \\
\hline $\begin{array}{l}\text { Distribution Coefficient } \\
\text { Selectivity Coefficient }\end{array}$ & 1.927 & 0.929 \\
\hline
\end{tabular}




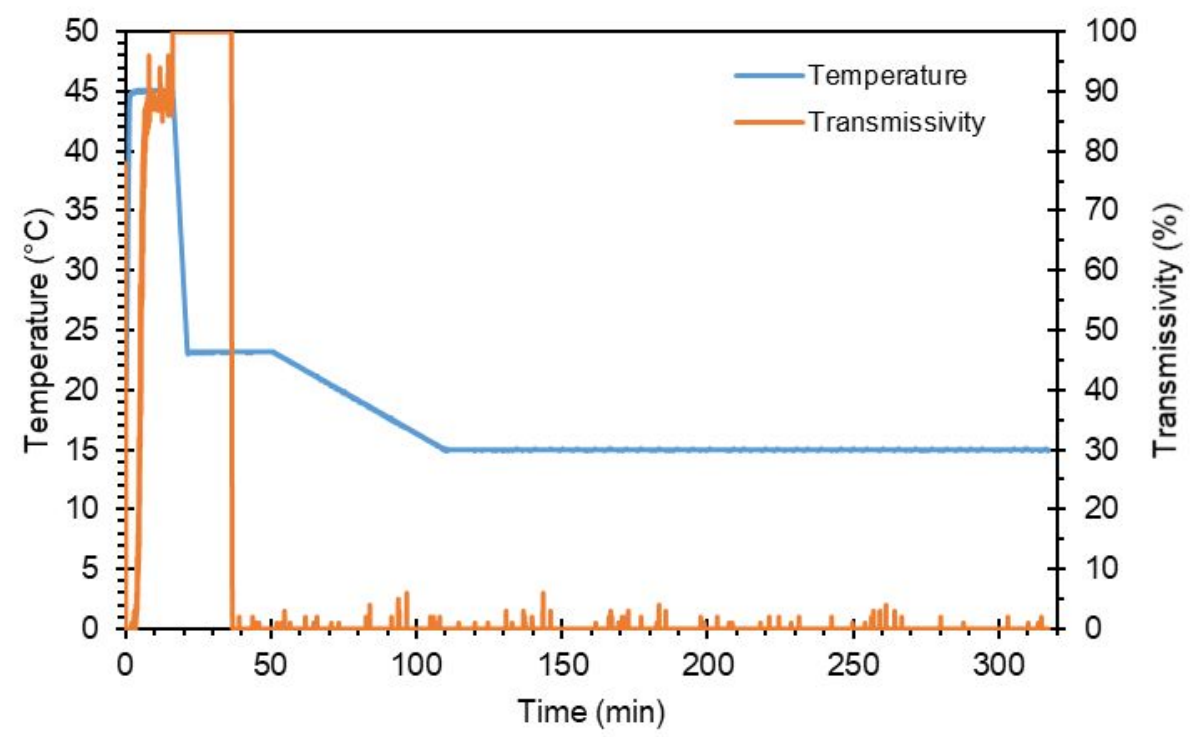

Figure S5 Temperature and transmissivity profile for fenofibrate-impurity E crystallisation. Time to crystallisation was $14 \mathrm{~min} 47 \mathrm{sec}$

Table $\mathbf{S 6}$ Calculation of maximum selectivity value for paracetamol and acetanilide case study.

\begin{tabular}{|c|c|c|}
\hline & Paracetamol & Acetanilide \\
\hline Molecular Formula & $\mathrm{C}_{8} \mathrm{H}_{9} \mathrm{NO}_{2}$ & $\mathrm{C}_{8} \mathrm{H}_{9} \mathrm{NO}$ \\
\hline Molecular Weight & $151.163 \mathrm{~g} \mathrm{~mol}^{-1}$ & $135.166 \mathrm{~g} \mathrm{~mol}^{-1}$ \\
\hline$X f$ & 0.952 & 0.048 \\
\hline Xs & 0.998 & 0.002 \\
\hline $\mathbf{X I}$ & 0.860 & 0.141 \\
\hline Distribution Coefficient & 1.116 & 0.163 \\
\hline Selectivity Coefficient & \multicolumn{2}{|c|}{$\alpha=0.012$} \\
\hline
\end{tabular}

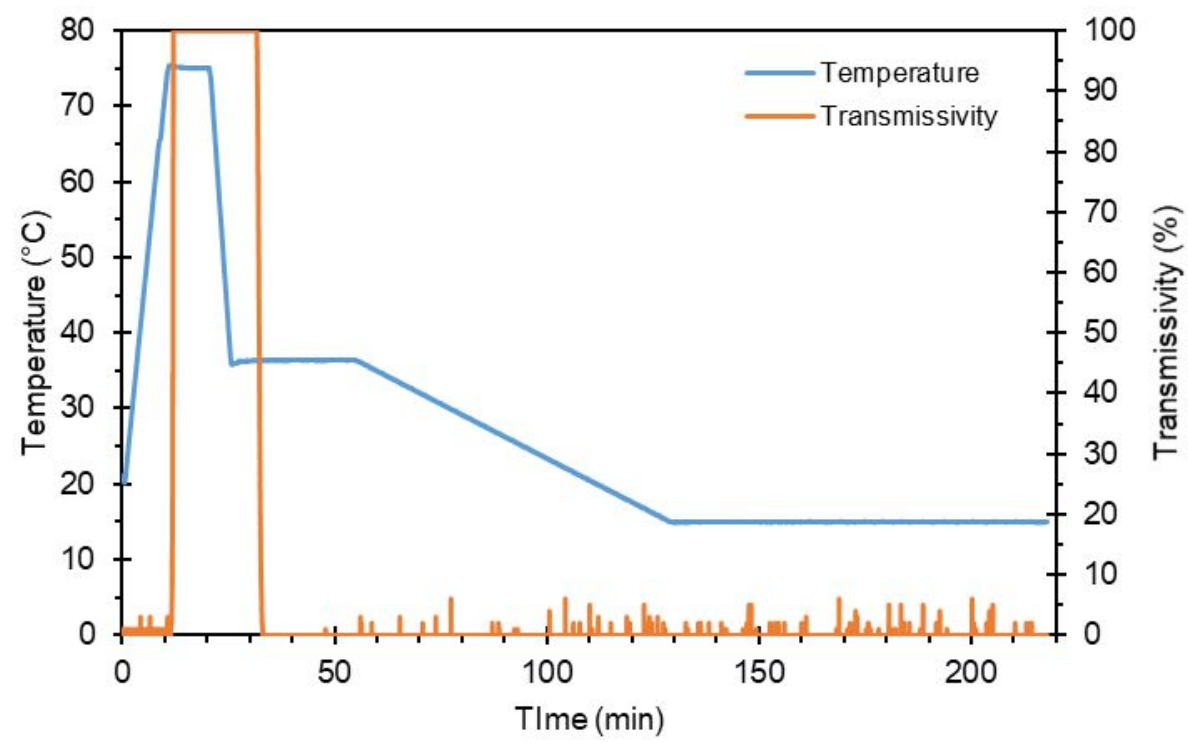

Figure S6 Temperature and transmissivity profile for paracetamol-acetanilide crystallisation. Time to crystallisation was $7 \mathrm{~min} 25 \mathrm{se}$ 
Table S7 Calculation of maximum selectivity value for paracetamol and metacetamol case study.

\begin{tabular}{ccc}
\hline & Paracetamol & Metacetamol \\
\hline Molecular Formula & $\mathrm{C}_{8} \mathrm{H}_{9} \mathrm{NO}_{2}$ & $\mathrm{C}_{8} \mathrm{H}_{9} \mathrm{NO}_{2}$ \\
Molecular Weight & $151.163 \mathrm{~g} \mathrm{~mol}^{-1}$ & $151.163 \mathrm{~g} \mathrm{~mol}^{-1}$ \\
$\boldsymbol{X}_{\boldsymbol{f}}$ & 0.961 & 0.039 \\
$\boldsymbol{X}_{\boldsymbol{s}}$ & 0.995 & 0.005 \\
$\boldsymbol{X}_{\boldsymbol{I}}$ & 0.893 & 0.107 \\
Distribution Coefficient & 1.114 & 0.119 \\
Selectivity Coefficient & & $\mathbf{\alpha = 0 . 0 4 2}$ \\
\hline
\end{tabular}

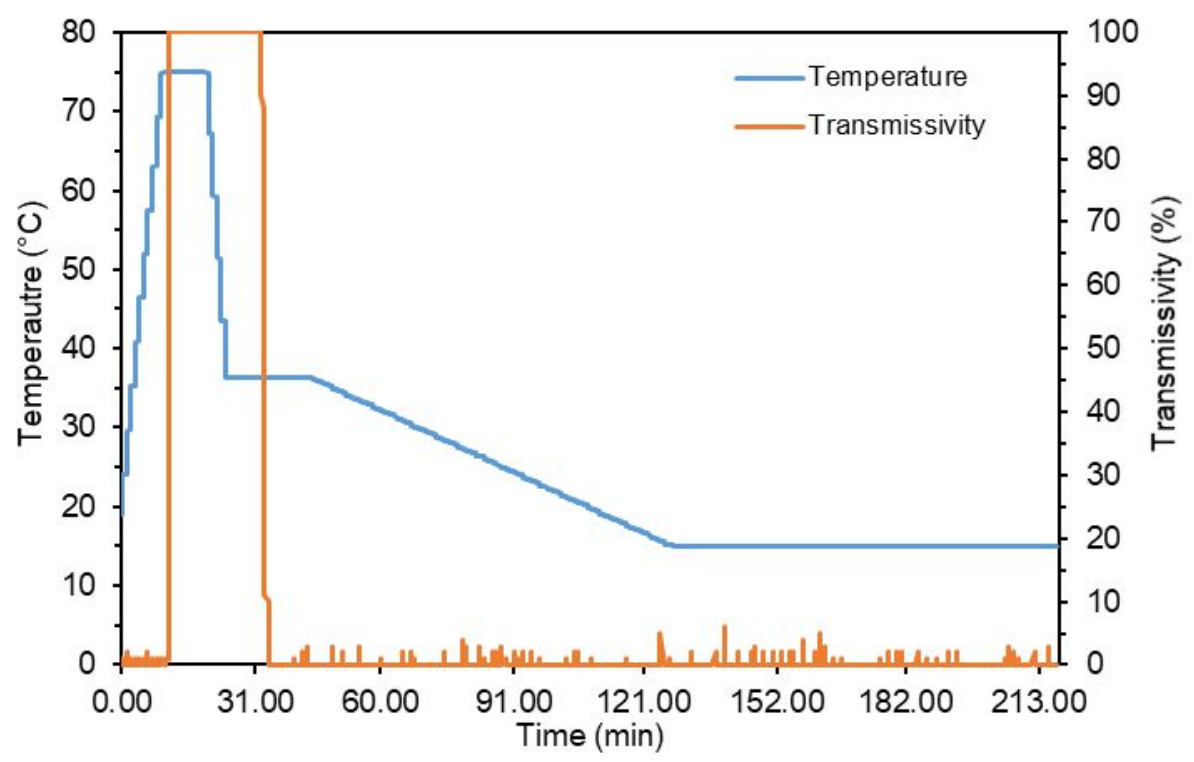

Figure S7 Temperature and transmissivity profile for paracetamol-metacetamol crystallisation. Time to crystallisation was $8 \mathrm{~min} 13 \mathrm{sec}$

Table S8 Comparison of product recovery from crystallisations. ${ }^{\text {BBased }}$ on literature reported solubility. ${ }^{\mathrm{b}} \mathrm{After}$ filtration, washing and drying.

\begin{tabular}{ccccc}
\hline API & Impurity & $\begin{array}{c}\text { Maximum Mass of } \\
\text { Product }^{\mathbf{a}}(\mathbf{m g})\end{array}$ & $\begin{array}{c}\text { Amount of Product } \\
\text { Isolated }^{\mathbf{b}}(\mathbf{m g})\end{array}$ & Recovery (\%) \\
\hline Paracetamol & Acetanilide & 672.5 & 534.9 & 79.5 \\
Paracetamol & Metacetamol & 670.5 & 604.9 & 90.2 \\
Fenofibrate & Fenofibric Acid & 332.9 & 290.0 & 87.1 \\
Fenofibrate & Impurity E & 328.4 & 292.4 & 89.0 \\
\hline
\end{tabular}


(a)

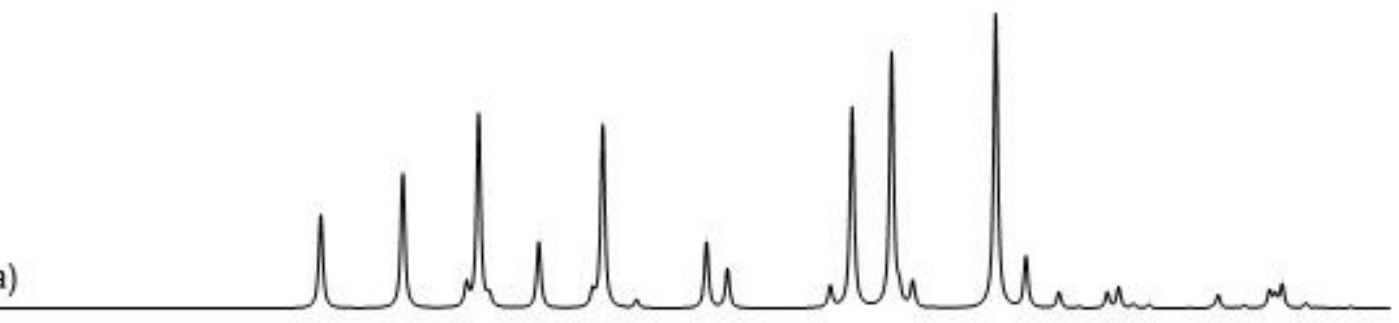

(b)

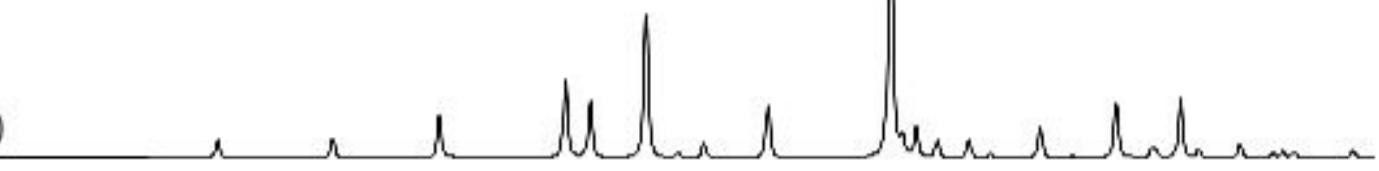

(c)

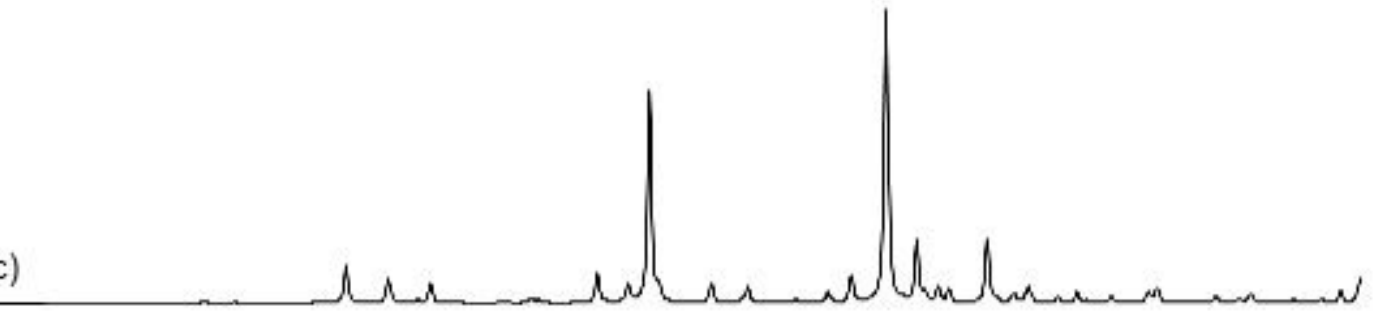

(d)
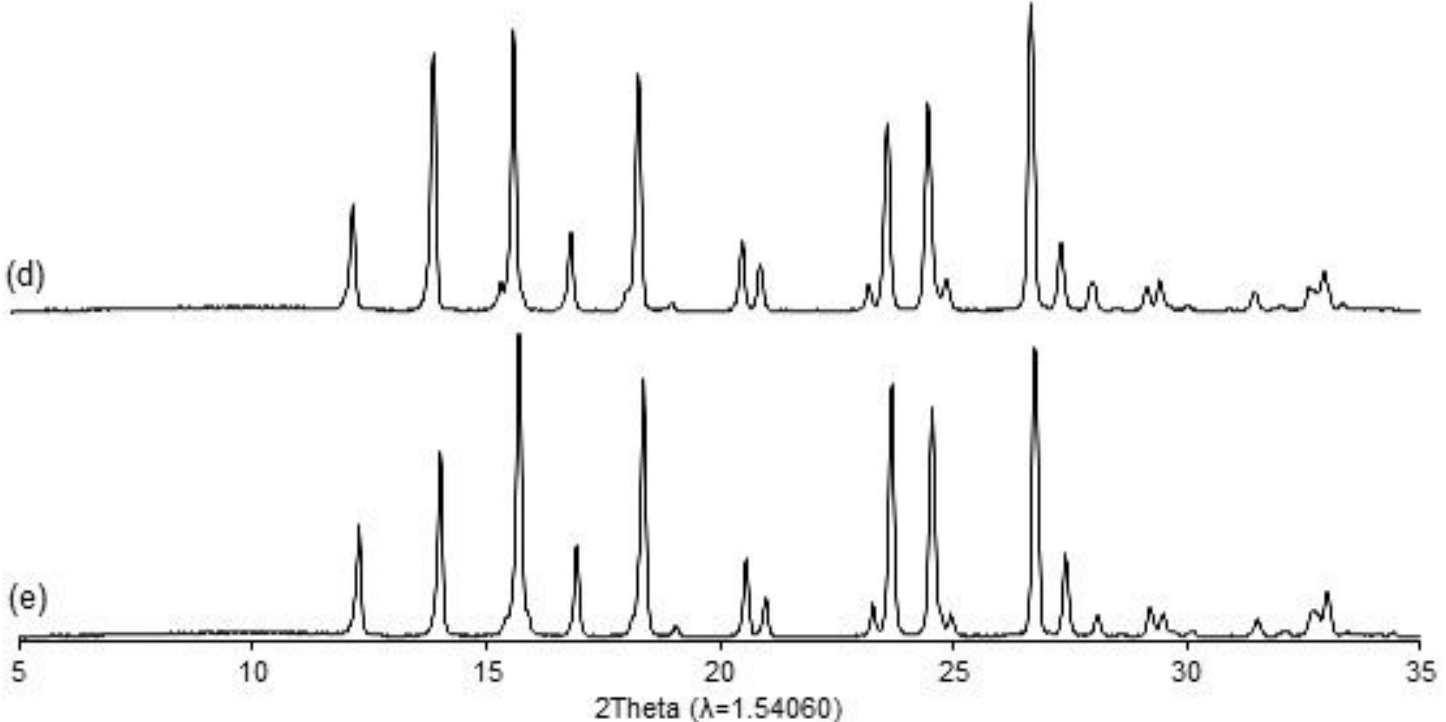

Figure S8 XRPD patterns of (a) paracetamol form I calculated ${ }^{3}$ (b) paracetamol form II calculated ${ }^{4}$ (c) paracetamol form III calculated ${ }^{5}(\mathrm{~d})$ paracetamol contaminated with acetanilide $(0.8 \mathrm{~mol} \%)$ and $(\mathrm{e})$ paracetamol contaminated with metacetamol $(2.5 \mathrm{~mol} \%)$ 
(a)

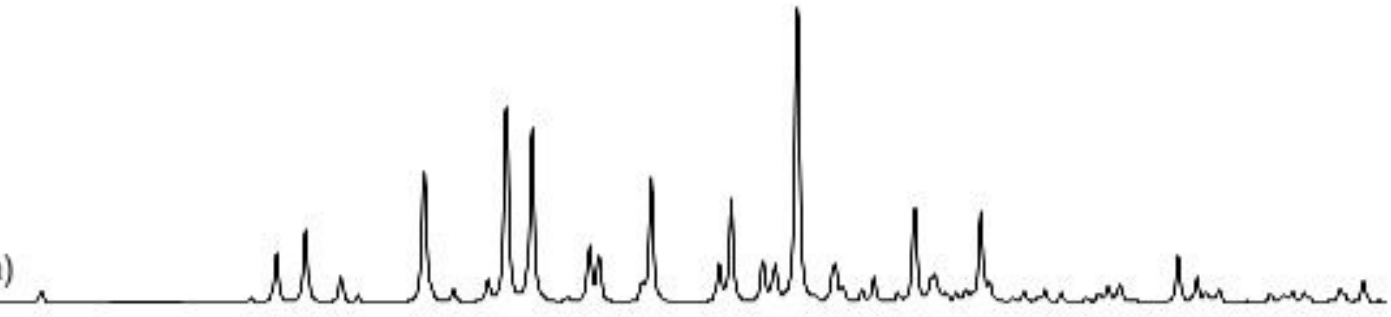

(b)

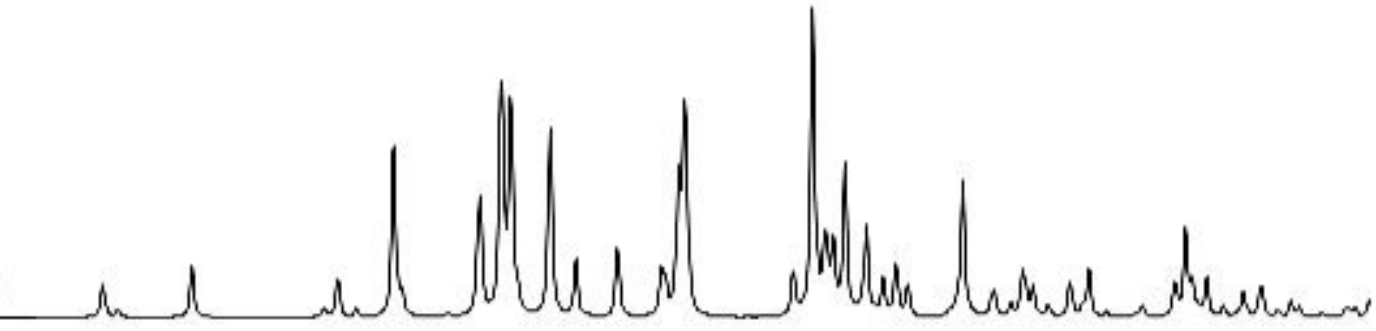

(c)
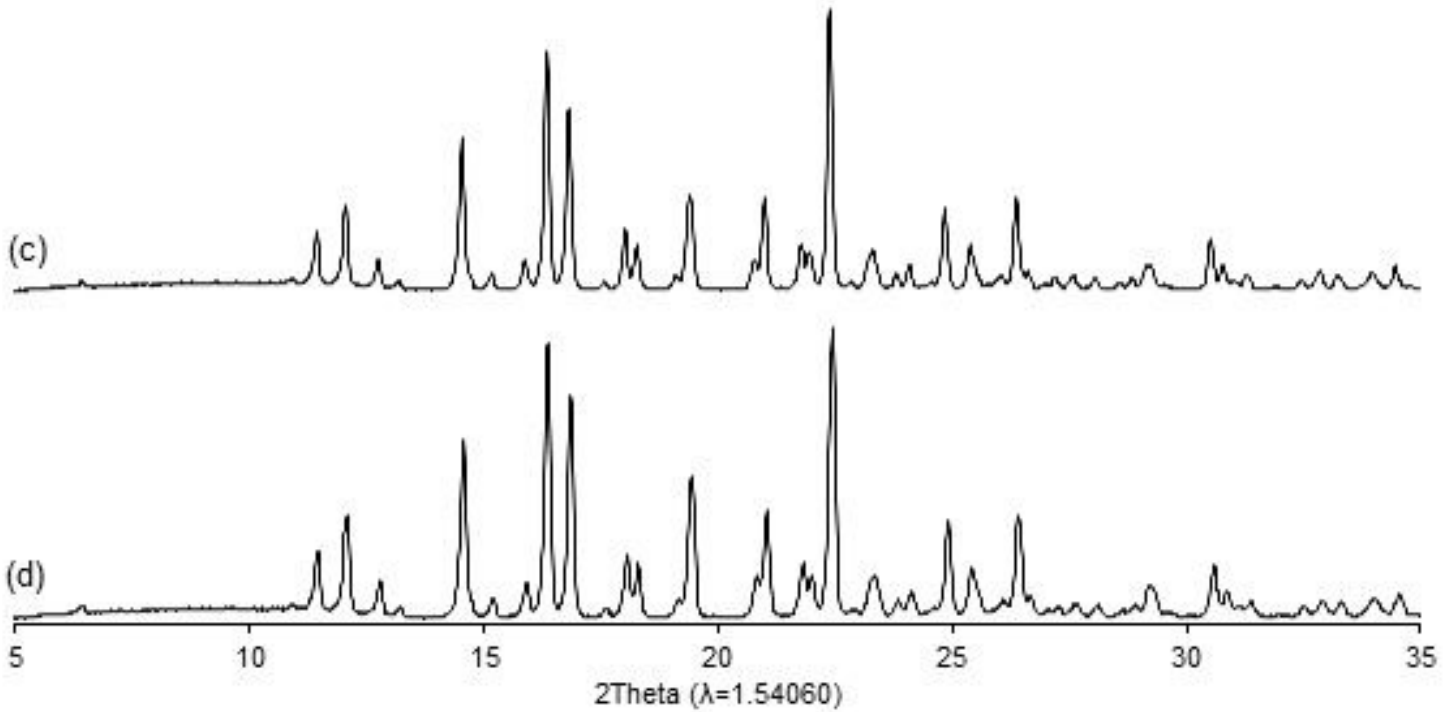

Figure S9 XRPD patterens of (a) fenofibrate form I calculated ${ }^{6}$ (b) fenofibrate form II calculated ${ }^{7}$ (c) fenofibrate contaminated with fenofibric acid (1.5 mol\%) (d) fenofibrate contaminated with impurity E (6.4 mol\%) 


\section{Impurity Mapping}

Table S9 Experimental data for stepwise dissolution of fenofibrate crystals contaminated with impurity E.

\begin{tabular}{cccc}
\hline $\begin{array}{c}\text { No. of Solvent } \\
\text { Portions }\end{array}$ & $\begin{array}{c}\text { Approx. \% } \\
\text { Solid Dissolved }\end{array}$ & $\begin{array}{c}\text { FEN Peak Area } \\
\%\end{array}$ & $\begin{array}{c}\text { IPE Peak Area } \\
\%\end{array}$ \\
\hline 0 & 0.0 & 94.65 & 5.35 \\
1 & 12.7 & 95.02 & 4.98 \\
2 & 25.3 & 89.49 & 10.51 \\
3 & 37.9 & 88.92 & 11.08 \\
4 & 50.6 & 88.17 & 11.83 \\
5 & 63.2 & 86.32 & 13.68 \\
6 & 75.9 & 86.42 & 13.58 \\
7 & 88.5 & 87.42 & 12.58 \\
8 & 100.0 & 88.09 & 11.91 \\
\hline
\end{tabular}

Table S10 Experimental data for stepwise dissolution of paracetamol crystals contaminated with metacetamol.

\begin{tabular}{cccc}
$\begin{array}{c}\text { No. of Solvent } \\
\text { Portions }\end{array}$ & $\begin{array}{c}\text { Approx. \% } \\
\text { Solid Dissolved }\end{array}$ & $\begin{array}{c}\text { PCM Peak Area } \\
\%\end{array}$ & $\begin{array}{c}\text { MET Peak Area } \\
\%\end{array}$ \\
\hline 0 & 0.0 & 99.30 & 0.70 \\
1 & 10.5 & 99.03 & 0.97 \\
2 & 21.0 & 98.95 & 1.05 \\
3 & 31.5 & 98.79 & 1.21 \\
4 & 42.1 & 98.71 & 1.29 \\
5 & 52.6 & 98.62 & 1.38 \\
6 & 63.1 & 98.55 & 1.45 \\
7 & 73.6 & 98.54 & 1.46 \\
8 & 84.1 & 98.53 & 1.47 \\
9 & 94.6 & 98.53 & 1.47 \\
10 & 100.0 & 98.65 & 1.35 \\
\hline
\end{tabular}




\section{Solid State Behaviour}

The phase diagram for each API-impurity binary mixture is modelled using the rearranged Schroeder van Laar equation (1) where the mole fraction of a component $X_{\text {component }}$ is related to the enthalpy of fusion $\Delta H_{\text {fus }}$, universal gas constant $R$, melting point of the mixture $T$ and pure component melting temperature $T_{m}$. Values for thermodynamic parameters were experimentally measured using differential scanning calorimetry (DSC) where values were not available in the literature. When plotted as $T$ versus $X_{\text {compnonent, }}$ the intersection of the API and impurity curves gives the predicted eutectic composition and melting temperature.

$$
\frac{1}{T}=\frac{1}{T_{m}}-\frac{R \operatorname{LnX} X_{\text {component }}}{\Delta H_{\text {fus }}}
$$
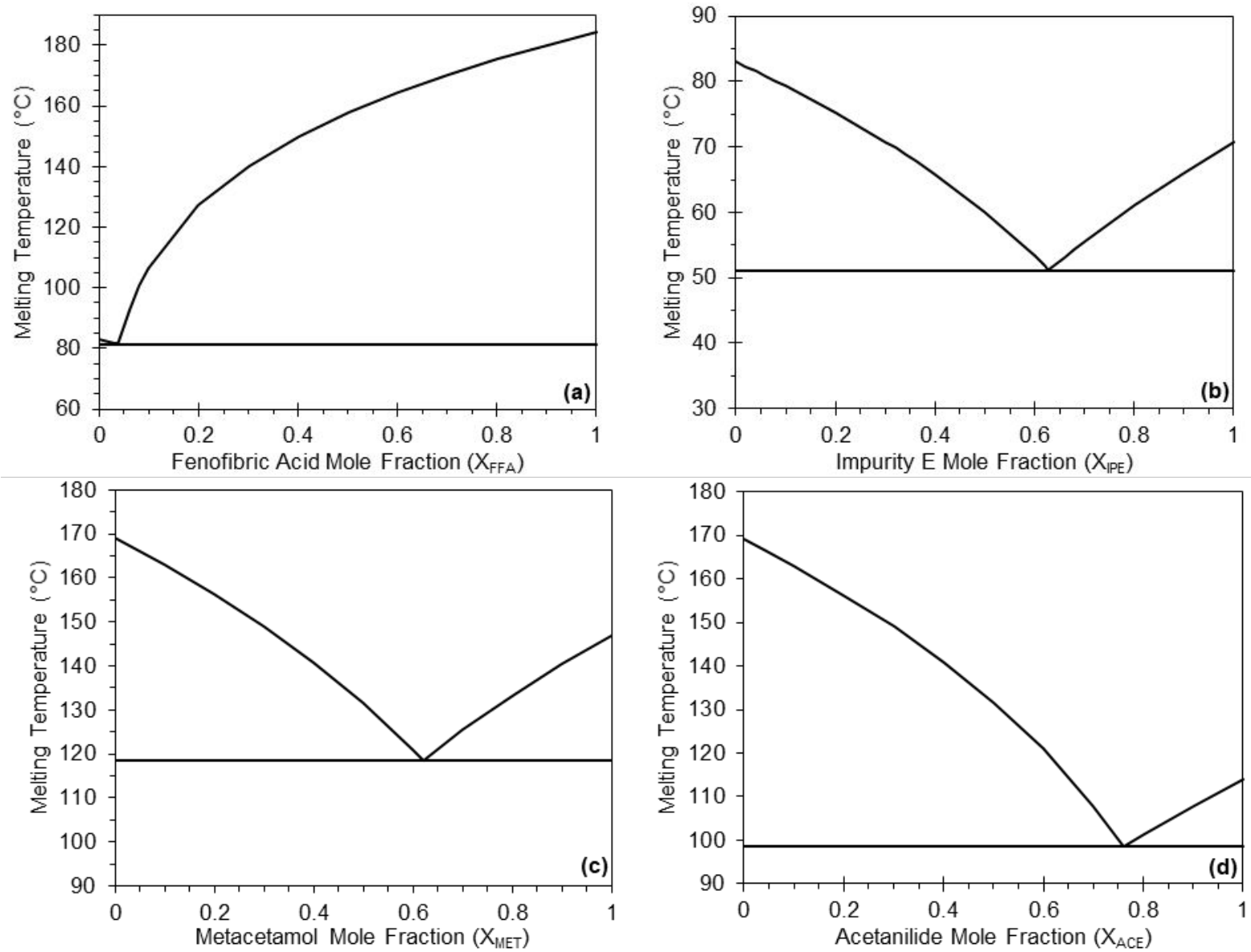

Figure S10 Predicted binary phase diagrams for the case studies in this work. (a) Fenofibrate-fenofibric acid, predicted eutectic $X_{F F A}=0.038, T_{m}=81.4^{\circ} \mathrm{C}$. (b) Fenofibrate-impurity $E$, predicted eutectic $X_{\text {IPE }}=0.629, T_{m}=51.1$ ${ }^{\circ} \mathrm{C}$. (c) Paracetamol-metacetamol, predicted eutectic $X_{M E T}=0.620, T_{m}=118.6{ }^{\circ} \mathrm{C}$. (d) Paracetamol-acetanilide, predicted eutectic $\mathrm{X}_{\mathrm{ACE}}=0.760, \mathrm{~T}_{\mathrm{m}}=98.6^{\circ} \mathrm{C}$. 
Table S11 Data extracted from DSC measurements of fenofibrate-impurity E crystallised mixtures, used to construct the binary phase diagram.

\begin{tabular}{cccc}
\hline $\mathbf{X}_{\mathbf{I P E}}$ & Series & $\begin{array}{c}\text { Peak Maxima } \\
\left({ }^{\circ} \mathbf{C}\right)\end{array}$ & $\begin{array}{c}\text { Peak Area } \\
(\mathbf{J} / \mathbf{g})\end{array}$ \\
\hline 0.000 & $\mathrm{~A}$ & 83.1 & 81.0 \\
0.020 & $\mathrm{~B}$ & 81.6 & 86.8 \\
0.040 & $\mathrm{~B}$ & 80.8 & 85.3 \\
0.060 & $\mathrm{~B}$ & 81.1 & 82.4 \\
0.081 & $\mathrm{~B}$ & 81.1 & 79.8 \\
0.099 & $\mathrm{~A}$ & 79.8 & 80.9 \\
0.199 & $\mathrm{~A}$ & 78.5 & 75.7 \\
0.299 & $\mathrm{~A}$ & 74.4 & 68.6 \\
0.399 & $\mathrm{~A}$ & 69.5 & 64.7 \\
0.499 & $\mathrm{~A}$ & 65.6 & 65.9 \\
0.552 & $\mathrm{~B}$ & 68.5 & 87.7 \\
0.599 & $\mathrm{~A}$ & 61.8 & 62.6 \\
0.622 & $\mathrm{~B}$ & 64.1 & 72.6 \\
0.642 & $\mathrm{~B}$ & 64.5 & 72.6 \\
0.662 & $\mathrm{~B}$ & 64.0 & 51.4 \\
0.682 & $\mathrm{~B}$ & 64.4 & 60.7 \\
0.699 & $\mathrm{~A}$ & 59.9 & 49.1 \\
0.751 & $\mathrm{~B}$ & 62.8 & 58.5 \\
0.799 & $\mathrm{~A}$ & 60.4 & 54.9 \\
0.899 & A & 64.3 & 57.6 \\
1.000 & A & 72.1 & 62.0 \\
\hline
\end{tabular}


Table S12 Data extracted from DSC measurements of paracetamol-metacetamol crystallised mixtures, used to construct the binary phase diagram and Tamman triangle plot

\begin{tabular}{ccccc}
\hline $\mathbf{X}_{\text {MET }}$ & $\begin{array}{c}\text { Peak Maximum } \\
\left({ }^{\circ} \mathbf{C}\right)\end{array}$ & $\begin{array}{c}\text { Component } \\
\text { Peak Area } \\
(\mathbf{J} / \mathbf{g})\end{array}$ & $\begin{array}{c}\text { Eutectic (Horizontal Line) } \\
\text { Peak Maximum } \\
\left({ }^{\circ} \mathbf{C}\right)\end{array}$ & $\begin{array}{c}\text { Peak Area } \\
(\mathbf{J} / \mathbf{g})\end{array}$ \\
\hline 0.000 & 171.9 & 172.0 & -- & -- \\
0.002 & 171.2 & 212.0 & -- & -- \\
0.005 & 170.6 & 170.6 & -- & -- \\
0.010 & 168.0 & 159.4 & -- & -- \\
0.020 & 169.8 & 147.3 & 119.7 & -- \\
0.030 & 169.4 & 153.4 & -- & 1.6 \\
0.050 & 168.0 & 137.7 & 119.2 & 8.3 \\
0.096 & 164.0 & 112.7 & 120.1 & 27.1 \\
0.201 & 163.0 & 75.8 & 121.2 & 60.4 \\
0.300 & 150.4 & 73.9 & 123.0 & 93.3 \\
0.400 & 131.5 & 46.0 & 120.7 & 105.7 \\
0.500 & 136.0 & 1.3 & 120.0 & -- \\
0.600 & 124.2 & 143.4 & -- & 94.5 \\
0.650 & 124.1 & 155.2 & -- & 99.0 \\
0.700 & 117.9 & 94.5 & 117.9 & 29.1 \\
0.800 & 132.9 & 47.2 & 120.9 & 33.6 \\
0.900 & 146.0 & 126.2 & 119.5 & 16.2 \\
0.920 & 145.1 & 121.4 & 120.9 & 5.5 \\
0.940 & 145.3 & 100.4 & 119.6 & -- \\
0.960 & 147.5 & 117.1 & 108.9 & -- \\
0.985 & 148.7 & 141.9 & -- & -- \\
0.990 & 149.4 & 148.6 & -- & - \\
0.995 & 148.8 & 152.9 & -- & - \\
0.998 & 148.5 & 151.5 & -- & - \\
1.000 & 149.2 & 170.7 & & - \\
\hline
\end{tabular}

\section{References}

(1) Granberg, R. A.; Rasmuson, Å. C. Solubility of Paracetamol in Pure Solvents. J. Chem. Eng. Data 1999, 44, 1391-1395. https://doi.org/10.1021/je990124v.

(2) Watterson, S.; Hudson, S.; Svärd, M.; Rasmuson, A. C. Thermodynamics of Fenofibrate and Solubility in Pure Organic Solvents. Fluid Phase Equilib. 2014, 367, 143-150. https://doi.org/10.1016/j.fluid.2014.01.029.

(3) Haisa, M.; Kashino, S.; Maeda, H. The Orthorhombic Form of P-Hydroxyacetanilide. Acta Crystallogr. Sect. B 1974, 2510-2512.

(4) Nichols, G.; Frampton, C. S. Physicochemical Characterization of the Orthorhombic Polymorph of Paracetamol Crystallized from Solution. J. Pharm. Sci. 1998, 87, 684693. https://doi.org/10.1021/js970483d.

(5) Perrin, M.; Neumann, M. A.; Zaske, L. Crystal Structure Determination of the Elusive Paracetamol Form III. Chem. Commun. 2009, 3181-3183. https://doi.org/10.1039/b822882e.

(6) Henry, R. F.; Zhang, G. Z.; Gao, Y.; Buckner, I. S. Fenofibrate. Acta Crystallogr. Sect. E 2003, 59, 699-700. https://doi.org/10.1107/S1600536803008274.

(7) Balendiran, G. K.; Rath, N.; Kotheimer, A.; Miller, C.; Zeller, M.; Rath, N. P. Biomolecular Chemistry of Isopropyl Fibrates. J. Pharm. Sci. 2012, 101 (4), 15551569. https://doi.org/10.1002/jps.23040. 